\title{
Effects of leg muscle fatigue on gait in patients with Parkinson's disease and controls with high and low levels of daily physical activity
}

\author{
Paulo Cezar Rocha Santos ${ }^{a, *}$, Lilian Teresa Bucken Gobbi ${ }^{a}$, Diego Orcioli-Silva ${ }^{a}$, \\ Lucas Simieli ${ }^{\mathrm{a}}$, Jaap H. van Dieën ${ }^{\mathrm{b}}$, Fabio Augusto Barbieri ${ }^{\mathrm{a}, \mathrm{c}}$ \\ ${ }^{a}$ UNESP, Univ Estadual Paulista at Rio Claro - LEPLO, São Paulo, Brazil \\ ${ }^{\mathrm{b}}$ MOVE Research Institute Amsterdam, Faculty of Human Movement Sciences, VU University Amsterdam, Amsterdam, The Netherlands \\ ${ }^{\mathrm{c}}$ UNESP, Univ Estadual Paulista at Bauru - UNESP - LIVIA, São Paulo, Brazil
}

\section{A R T I C L E IN F O}

\section{Article history:}

Received 26 September 2015

Received in revised form 17 March 2016

Accepted 1 April 2016

\section{Keywords:}

Parkinson's disease

Fatigue

Gait impairments

Physical activity levels

\begin{abstract}
A B S T R A C T
Patients with Parkinson's disease (PD) are more susceptible to muscle fatigue, which can damage their gait. Physical activity can improve muscle condition, which is an important aspect during walking. The aim of this study was to analyze the effects of lower limb muscle fatigue on gait in patients with PD and healthy individuals, grouped according to physical activity level. Twenty Patients with PD (PD group) and 20 matched individuals (control group) were distributed according to physical activity level into four subgroups of ten individuals (active and inactive). Participants performed three walking trials before and after lower limb muscle fatigue, induced by a repeated sit-to-stand task on a chair. Kinematic (stride length, width, duration, velocity and percentage of time in double support) and kinetic (propulsive and breaking anterior-posterior and medio-lateral impulse) gait parameters were analyzed. In both groups, participants increased stride length and velocity and decreased stride duration and braking vertical impulse after lower limb muscle fatigue. The PD groups presented higher step width and percentage of double time support than the control groups before muscle fatigue. The control groups increased step width and decreased percentage of time in double support, while the PD groups did not change these parameters. For physical activity level, active individuals presented longer stride length, greater stride velocity, higher braking and propulsive anterior-posterior impulse and shorter step width than inactive individuals. Groups sought more balance and safety after lower limb muscle fatigue. Physical activity level does not appear to modify the effects of lower limb muscle fatigue during unobstructed walking in individuals with PD or controls.
\end{abstract}

๑) 2016 Published by Elsevier B.V.

\section{Introduction}

The general increase in human life expectancy coincides with an increase in the number of people with chronic diseases, such as Parkinson's disease (PD). Patients with PD present several motor and non-motor impairments [1], including a higher perception of fatigue than age-matched controls [2]. The exacerbated perception of fatigue is caused by musculoskeletal and neurophysiological impairments associated with $\mathrm{PD}$, particularly resulting from altered norepinephrine and serotonin production due to degeneration of neurons of the raphe nuclei and locus coeruleus

\footnotetext{
* Corresponding author at: Posture and Gait Studies Lab, Department of Physical Education, UNESP, Univ Estadual Paulista at Rio Claro, Avenida 24-A, 1515, Bela Vista, Rio Claro/São Paulo, Brazil. Tel.: +55 19981715558.

E-mail address: paulocezarr@hotmail.com (P.C.R. Santos).
}

$[1,3]$. However, little is known about the effects of fatigue on movement in patients with PD. Previous studies indicate that gait is affected by fatigue in an age-dependent manner [4]. Gait adjustments with muscle fatigue are more pronounced in individuals over 40 years of age than in younger individuals. These adjustments appear to be aimed at maintaining adequate control of balance in the fatigued condition [4]. It is conceivable that individuals with PD demonstrate even more pronounced gait adjustments, since patients with PD present deficits in muscle strength and motor control [5].

Muscle fatigue could be expected to affect gait less in individuals with higher physical activity levels, due to their better neuromuscular and cardiovascular condition [6], which influences the process of muscle fatigue development [7]. However, the effects of muscle fatigue on gait in young adults are not dependent on their physical activity level [8]. This might be due to young adults having substantial remaining capacity to deal with the 
limited balance threat of normal unperturbed gait even with substantial muscle fatigue. Thus, whether this is generalized to older and diseased populations is unknown. Regular physical activity improves functional capacity [9] and walking ability [10] in patients with PD and likely slows down fatigue development, but whether it improves their gait in a fatigued state is unknown.

Therefore, the aim of the present study was to analyze the effects of lower limb muscle fatigue on gait in patients with PD and in healthy individuals, grouped according to physical activity level. We expected that patients with PD would be more affected by lower limb muscle fatigue than healthy controls. In addition, we hypothesized that both groups would improve anterior-posterior and medio-lateral balance control after lower limb muscle fatigue. Furthermore, we hypothesized that there would be an interaction between lower limb muscle fatigue and physical activity level, with greater effects of muscle fatigue on gait parameters in inactive participants of both groups.

\section{Methods}

\subsection{Participants}

After signing the informed consent, forty subjects participated in the study which had been approved by the local ethics committee (\#3083/2011); 20 individuals with PD (PD group), according to the UK Brain Bank Criteria [11], and 20 neurologically healthy matched-individuals (control group). Individuals were included if they met the following inclusion criteria: (i) independently living in the community, able to walk without the use of any aids, not presenting balance or vision disorders (to guarantee no interference from individual disorders, walking limitations or safety during walking); (ii) did not have diabetes, hypertension, cardio-respiratory diseases. In addition, for patients with PD, the individuals were included in the study if the stage in Hoehn \& Yahr Scale $(H \& Y)[12]$ was $\leq 3$. During the sample selection process, 19 patients with PD and 4 neurologically healthy individuals were initially recruited but did not fit the criteria of the study.

Within the groups, two sub-groups $(n=10)$ were formed, according to physical activity level (active and inactive group). The Modified Baecke Questionnaire for Older Adults (MBQOA) [13] was used to determine the physical activity level. The active group was composed of individuals who scored $\geq 5$ on the questionnaire and the inactive group was composed of individuals who scored $\leq 4$ [14]. The PD group and the control group were matched by gender, age, body height and body mass and these parameters were similar in the sub-groups. The PD sub-groups were matched according to the PD stages and the clinical characteristics. A neuropsychiatrist performed a clinical assessment of the patients with PD to determine the stage of the disease in each patient and to test them on the motor section of the Unified Parkinson's Disease Rating Scale (UPDRS) [15], H\&Y [12] and Mini-Mental State Examination (MMSE) [16].

\subsection{Maximum voluntary contraction protocol}

Maximum voluntary isometric contractions were performed on a leg press device $[4,9]$. A load cell with a precision of $0.98 \mathrm{~N}$ was used in combination with a signal amplifier (EMG System do Brasil Ltda.). The participants performed the test using both lower limbs simultaneously (hip joint angle $=110^{\circ}$ and knee joint angle $=90^{\circ}$ with $180^{\circ}$ as full extension) and were instructed to produce maximum force as fast as possible for $5 \mathrm{~s}$. Two attempts were performed before and after lower limb muscle fatigue, with a 2 min rest between attempts. The means of the two attempts before and after muscle fatigue were calculated for each participant. The maximum voluntary contraction was used to confirm the presence of muscle fatigue $[4,8]$.

\subsection{Lower limb muscle fatigue protocol}

To induce lower limb muscle fatigue, participants performed a repeated sit-to-stand task from a chair with arms across the chest region $[4,8]$. The frequency of the sit-to-stand movement was controlled by a metronome ( 30 cycles/min). A standard chair (43 cm in height, $41 \mathrm{~cm}$ in width and $42 \mathrm{~cm}$ in depth) without armrests was used for all participants. The instructions given to the participants were: stand up in an upright position with knees fully extended, then sit back down and repeat the task at the beat of the metronome until you can no longer perform the task. The fatigue protocol was stopped when one of the following conditions was met; the participant indicated their inability to continue, the movement frequency fell below and remained below 30 cycles/ min after encouragement, or after $30 \mathrm{~min}$. The time to fatigue was recorded. The gait task and subsequently the maximal voluntary protocol were repeated immediately after the fatigue protocol.

\subsection{Gait task}

Participants performed three trials of unobstructed gait before and after the lower limb muscle fatigue protocol. Participants received the instruction to walk over an $8 \mathrm{~m}$ wooden pathway, which was covered with a black rubber carpet ( $3 \mathrm{~mm}$ thick), at a self-selected speed. The gait parameters of the stride (period between two consecutive heel contacts of the right limb) in the middle of the pathway were analyzed in the study.

\subsection{Data analysis}

Acquisition of kinematic gait parameters was accomplished with a three-dimensional optoelectronic system (OPTOTRAK Certus - 3D Motion Measurement System - Northeim Digital NDI, Waterloo, Ontario, Canada), positioned in the right sagittal plane, using a sampling rate of 100 samples/s. Infrared emitters were placed over the lateral aspect of the calcaneus and the head of the fifth metatarsus of the right limb, and over the medial aspect of the calcaneus and the head of the first metatarsus of the left limb. Data were filtered using a 5th order low-pass digital Butterworth filter (zero-lag) with a cutoff frequency of $6 \mathrm{~Hz}$. Stride length, stride duration, stride velocity, percentage of time in double support and step width were calculated.

Ground reaction forces were measured using a force plate (AccuGait, Advanced Mechanical Technologies) with a sampling frequency of $200 \mathrm{~Hz}$, synchronized with the Optotrak system. The force plate was positioned across the central area of the pathway. The kinetic data were filtered using a 4th order low-pass digital Butterworth filter (zero-lag) with a cutoff frequency of $16 \mathrm{~Hz}$. The magnitude of the ground reaction force was normalized by body weight from an acquisition in the orthostatic position and braking and propulsive vertical and anterior-posterior impulses were calculated.

\subsection{Statistical analysis}

The analysis with $\mathrm{G}^{*}$ Power software showed that a sample size of at least 32 individuals ( 8 in each group) was needed for an $80 \%$ probability to detect a difference of $20 \%$ between the two groups for the primary outcome (stride velocity) with a type I error of 0.05 , based on previously published data [4]. The statistical analyses were performed with SPSS 18.0 for Windows ${ }^{\circledR}$. The level of significance was set at 5\% for all analyses. Physical activity level was compared between the PD and control groups using one-way ANOVA. Time to fatigue was compared between the four subgroups using a two-way ANOVA, with group and physical activity level (active and inactive sub-groups) as factors. The gait parameters and maximum voluntary isometric contractions were 
compared by MANOVAs with group, physical activity level and fatigue (before and after lower limb muscle fatigue) as factors, with repeated measures over the last factor. When the MANOVA revealed a main effect, univariate analyses were used to locate the differences.

\section{Results}

\subsection{Groups characteristics}

The characteristics of each group, score in physical activity level and time to fatigue of the lower limb muscles are presented in Table 1. There was no difference between the PD and control groups for physical activity level (active: $p<0.90$ and inactive: $p<0.61$ ). For the time to fatigue of the lower limb muscles, the ANOVA indicated main effects of group $\left(F_{1,36}=17.34, p<0.001\right)$ and physical activity level $\left(F_{1,36}=14.48, p<0.001\right)$, and an interaction between group and physical activity level $\left(F_{1,36}=9.94, p<0.003\right)$. The control group showed a longer time to fatigue than the PD group ( $p<0.001)$, the active group showed a longer time to fatigue that the inactive group $(p<0.001)$ and the interaction indicated a much longer time to fatigue in the active control group than in all the other groups $(p<0.001)$.

\subsection{Maximum voluntary contraction protocol}

Regarding maximum voluntary isometric contractions (Table 2), the MANOVA indicated main effects of group $\left(F_{1,36}=12.10, p<0.001\right)$ and fatigue $\left(F_{1,36}=10.13, p<0.003\right)$, but no effects of physical activity level $\left(F_{1,36}=0.05, p=0.81\right)$ and no interactions between factors $\left(F_{1,36}=0.14, p=0.71\right)$. For group, the univariate analyses indicated that the PD group had a lower maximum voluntary isometric contraction than the control group. For fatigue, there was a reduction in the maximum voluntary isometric contraction after the fatigue protocol when compared to before. It is important to highlight that all individuals decreased the value of maximum voluntary isometric contraction after lower limb muscle fatigue.

Table 1

Means and standard deviations of height, body weight, age, physical activity level score and time to fatigue for the quadriceps muscles for the active and inactive PD groups and the control group. In addition, means and standard deviations of clinical characteristics of the active and inactive PD groups.

\begin{tabular}{|c|c|c|c|c|}
\hline \multirow[t]{2}{*}{ Parameters } & \multicolumn{2}{|l|}{ PD group } & \multicolumn{2}{|l|}{ Control group } \\
\hline & Active & Inactive & Active & Inactive \\
\hline Height $(\mathrm{cm})$ & $165.5 \pm 8.8$ & $166.5 \pm 5.8$ & $165.2 \pm 7.3$ & $170.6 \pm 6.9$ \\
\hline $\begin{array}{l}\text { Body } \\
\quad \text { weight (kg) }\end{array}$ & $72.6 \pm 7.6$ & $73.1 \pm 10.1$ & $68.6 \pm 9.4$ & $78.7 \pm 12.8$ \\
\hline Age (years) & $67.0 \pm 5.2$ & $71.7 \pm 5.0$ & $67.5 \pm 6.5$ & $71.4 \pm 6.4$ \\
\hline $\begin{array}{l}\text { Physical } \\
\text { activity } \\
\text { level (score) }\end{array}$ & $7.9 \pm 2.0$ & $2.9 \pm 1.3$ & $9.1 \pm 2.0$ & $1.3 \pm 0.5$ \\
\hline $\begin{array}{l}\text { Time to } \\
\text { muscle } \\
\text { fatigue (s) }\end{array}$ & $107.6 \pm 39.5$ & $80.8 \pm 21.3$ & $408.6 \pm 234.5$ & $122.3 \pm 133.2$ \\
\hline H\&Y (score) & $2.0 \pm 0.2$ & $1.8 \pm 0.2$ & & \\
\hline $\begin{array}{l}\text { UPDRS III } \\
\text { (score) }\end{array}$ & $31.8 \pm 6.9$ & $29.1 \pm 6.7$ & & \\
\hline MMSE (score) & $28.7 \pm 1.6$ & $29.1 \pm 1.5$ & & \\
\hline
\end{tabular}

PD, Parkinson's disease; H\&Y, Hoehn and Yahr Scale; UPDRS, Unified Parkinson Disease Rating Scale.

\subsection{Gait parameters}

For the kinematic gait parameters (Table 2), there were main effects of group (Wilks' Lambda $=0.63, F_{5,32}=3.70, p<0.009$ ), fatigue (Lambda $=0.44, F_{5,32}=8.02, p<0.001$ ) and physical activity level (Wilks' Lambda $=0.65, F_{5,32}=3.51, p<0.006$ ) and an interaction between group and fatigue (Wilks' Lambda $=0.62, F_{5,32}=3.97$, $p<0.006$ ), but no interactions between physical activity level and any other parameters. For group, the univariate analyses indicated shorter stride length $\left(F_{1,36}=8.77, p<0.006\right)$, percentage of time in double support $\left(F_{1,36}=4.16, \quad p<0.04\right)$ and stride duration $\left(F_{1,36}=4.42, p<0.04\right)$ and larger step width $\left(F_{1,36}=4.48, p<0.04\right)$ for the PD group. For fatigue, individuals increased stride length $\left(F_{1,36}=20.91, p<0.001\right)$ and speed $\left(F_{1,36}=19.71, p<0.001\right)$ and decreased stride duration $\left(F_{1,36}=10.92, p<0.002\right)$ and braking vertical impulse $\left(F_{1,36}=2.56, p<0.02\right)$ after lower limb muscle fatigue. For physical activity level, active individuals demonstrated longer stride length $\left(F_{1,36}=7.70, p<0.009\right)$, greater stride velocity $\left(F_{1,36}=5.52, p<0.02\right)$, higher braking $\left(F_{1,36}=4.51, p<0.04\right)$ and propulsive anterior-posterior impulse $\left(F_{1,36}=7.72, p<0.009\right)$ and shorter step width $\left(F_{1,36}=5.82, p<0.02\right)$ than inactive individuals.

Concerning the interaction between fatigue and group for gait parameters (Fig. 1), the analyses indicated significant differences for step width $\left(F_{1,36}=4.51, p<0.04\right)$ and percentage of time in double support $\left(F_{1,36}=7.28, p<0.01\right)$. The post hoc analyses identified that, before lower limb muscle fatigue, the PD group showed greater step width and a higher percentage of time in double support than the control group $(p=0.02)$. However, after muscle fatigue, the control group increased step width $(p=0.04)$ and decreased the percentage of time in double support $(p=0.005)$, while the PD group did not change these parameters $(p=0.32$ and $p=0.19$, respectively). As a result, after lower limb muscle fatigue, there was no difference in step width between the groups ( $p=0.27$ ), while the healthy controls presented a shorter double support time $(p=0.02)$ than the PD group.

\section{Discussion}

The aim of this study was to analyze the effects of lower limb muscle fatigue on gait in patients with PD and neurologically healthy individuals according to their physical activity level. Lower limb muscle fatigue caused adjustments in gait parameters in both the PD and control groups, which was in agreement in part with our hypothesis. However, in contrast with our expectations, the PD group did not demonstrate stronger effects of lower limb muscle fatigue; instead the gait changes were generally more pronounced in the healthy control group, even though the PD group had lower maximum voluntary isometric contraction strength. In contrast with our third hypothesis, we did not find interactions between physical activity level and lower limb muscle fatigue in gait parameters. Active and inactive individuals from both groups presented similar walking behavior after lower limb muscle fatigue. Active control individuals demonstrated longer time to fatigue of the lower limb muscles in comparison with the other groups. In the following paragraphs, we will discuss interpretations of the gait changes observed and explanations for the unexpected direction of the interaction between fatigue and PD, with larger fatigue-related changes in the control group compared to the PD group, as well as for the lack of interaction between physical activity level and fatigue.

Both PD and healthy subjects presented fatigue-related gait changes, which is consistent with an attempt to enhance the control of balance in the anterior-posterior direction. Similar changes in spatial-temporal gait parameters have been shown previously after gait perturbation, such as muscle fatigue $[4,8]$. An increase in stride length and a decrease in stride duration may 
Table 2

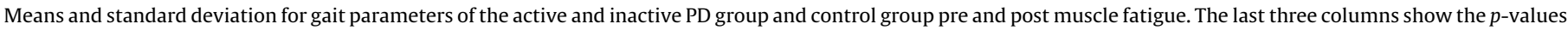
for main effects of group, fatigue and physical activity level, respectively.

\begin{tabular}{|c|c|c|c|c|c|c|c|c|}
\hline & & \multicolumn{2}{|l|}{ PD group } & \multicolumn{2}{|l|}{ Control group } & \multicolumn{3}{|c|}{ Main effects ( $p$-value) } \\
\hline & & Pre fatigue & Post fatigue & Pre fatigue & Post fatigue & Group & Fatigue & $\begin{array}{l}\text { Physical } \\
\text { activity }\end{array}$ \\
\hline \multirow{2}{*}{$\begin{array}{l}\text { Maximum voluntary } \\
\text { contraction protocol }(N)\end{array}$} & Active & $1785.56 \pm 559.58$ & $1592.89 \pm 403.67$ & $2576.03 \pm 623.67$ & $2445.98 \pm 553.50$ & \multirow[t]{2}{*}{0.001} & \multirow[t]{2}{*}{0.003} & \multirow[t]{2}{*}{0.812} \\
\hline & Inactive & $1762.04 \pm 461.0$ & $1696.48 \pm 575.26$ & $2402.67 \pm 1026.75$ & $2293.98 \pm 885.63$ & & & \\
\hline \multirow[t]{2}{*}{ Stride length $(\mathrm{cm})$} & Active & $124.69 \pm 10.69$ & $127.96 \pm 12.19$ & $132.28 \pm 10.69$ & $135.96 \pm 12.61$ & \multirow[t]{2}{*}{0.006} & \multirow[t]{2}{*}{0.001} & \multirow[t]{2}{*}{0.009} \\
\hline & Inactive & $107.57 \pm 15.21$ & $110.43 \pm 14.67$ & $123.27 \pm 14.93$ & $130.74 \pm 20.41$ & & & \\
\hline \multirow[t]{2}{*}{ Step width $(\mathrm{cm})$} & Active & $11.61 \pm 2.44$ & $11.33 \pm 2.21$ & $10.18 \pm 2.77$ & $11.52 \pm 2.90$ & \multirow[t]{2}{*}{0.04} & \multirow[t]{2}{*}{0.38} & \multirow[t]{2}{*}{0.02} \\
\hline & Inactive & $14.88 \pm 5.32$ & $14.14 \pm 1.99$ & $11.13 \pm 1.34$ & $12.29 \pm 2.20$ & & & \\
\hline \multirow{2}{*}{$\begin{array}{c}\text { Percentage of time in } \\
\text { double support (\%) }\end{array}$} & Active & $29.85 \pm 2.23$ & $28.94 \pm 1.69$ & $32.45 \pm 3.88$ & $26.02 \pm 1.45$ & \multirow[t]{2}{*}{0.04} & \multirow[t]{2}{*}{0.14} & \multirow[t]{2}{*}{0.11} \\
\hline & Inactive & $35.81 \pm 8.20$ & $39.31 \pm 12.81$ & $29.85 \pm 2.26$ & $27.11 \pm 3.77$ & & & \\
\hline \multirow[t]{2}{*}{ Stride duration (s) } & Active & $0.99 \pm 0.11$ & $0.98 \pm 0.10$ & $1.11 \pm 0.08$ & $1.06 \pm 0.12$ & \multirow[t]{2}{*}{0.04} & \multirow[t]{2}{*}{0.002} & \multirow[t]{2}{*}{0.27} \\
\hline & Inactive & $1.09 \pm 0.09$ & $1.02 \pm 0.13$ & $1.10 \pm 0.12$ & $1.06 \pm 0.06$ & & & \\
\hline \multirow[t]{2}{*}{ Stride velocity $(\mathrm{cm} / \mathrm{s})$} & Active & $122.17 \pm 17.55$ & $132.26 \pm 22.85$ & $119.75 \pm 14.15$ & $130.65 \pm 19.05$ & \multirow[t]{2}{*}{0.43} & \multirow[t]{2}{*}{0.001} & \multirow[t]{2}{*}{0.02} \\
\hline & Inactive & $99.57 \pm 18.53$ & $110.89 \pm 20.32$ & $113.37 \pm 20.87$ & $119.13 \pm 26.45$ & & & \\
\hline \multirow{2}{*}{$\begin{array}{l}\text { Braking vertical } \\
\text { impulse (\%BW/ms) }\end{array}$} & Active & $0.27 \pm 0.02$ & $0.27 \pm 0.05$ & $0.29 \pm 0.03$ & $0.28 \pm 0.03$ & \multirow[t]{2}{*}{0.76} & \multirow[t]{2}{*}{0.02} & \multirow[t]{2}{*}{0.14} \\
\hline & Inactive & $0.32 \pm 0.05$ & $0.29 \pm 0.05$ & $0.28 \pm 0.03$ & $0.28 \pm 0.04$ & & & \\
\hline \multirow{2}{*}{$\begin{array}{l}\text { Propulsive vertical } \\
\text { impulse (\%BW/ms) }\end{array}$} & Active & $0.24 \pm 0.04$ & $0.24 \pm 0.04$ & $0.28 \pm 0.03$ & $0.26 \pm 0.03$ & \multirow[t]{2}{*}{0.73} & \multirow[t]{2}{*}{0.18} & 0.56 \\
\hline & Inactive & $0.27 \pm 0.05$ & $0.28 \pm 0.05$ & $0.26 \pm 0.05$ & $0.25 \pm 0.05$ & & & \\
\hline Braking anterior-posterior & Active & $-0.026 \pm 0.005$ & $-0.027 \pm 0.006$ & $-0.029 \pm 0.005$ & $-0.032 \pm 0.003$ & 0.08 & 0.53 & 0.04 \\
\hline impulse $(\% \mathrm{BW} / \mathrm{ms})$ & Inactive & $-0.024 \pm 0.008$ & $-0.023 \pm 0.005$ & $-0.027 \pm 0.008$ & $-0.025 \pm 0.004$ & & & \\
\hline Propulsive anterior-posterior & Active & $0.026 \pm 0.007$ & $0.027 \pm 0.005$ & $0.032 \pm 0.003$ & $0.031 \pm 0.004$ & 0.35 & 0.09 & 0.009 \\
\hline impulse (\%BW/ms) & Inactive & $0.024 \pm 0.003$ & $0.025 \pm 0.005$ & $0.025 \pm 0.004$ & $0.026 \pm 0.005$ & & & \\
\hline
\end{tabular}

MF, muscle fatigue; PD, Parkinson's disease; BW, body weight.

facilitate balance control in the forward direction [17,18], albeit at the cost of increasing the risk of falling backward [19]. An increase in stride length $(\sim 3 \mathrm{~cm})$ is clinically relevant for patients with PD; a small increase in stride length was observed in previous studies $[4,8]$. First, longer strides require more muscle activation, which could be a problem due to weakness in this population [5]. Second, any increase in stride length, even small, can enhance balance control in the sagittal plane. In walking, the anterior margin of safety is negative, which could cause a forward fall [20]. An increase in stride length may decrease the magnitude of the negative margin and, consequently, the risk of forward falls [21]. Therefore, under the fatigued muscle condition, small adjustments are clinically important to avoid falls during gait.

The combination of modulation in stride length and stride duration causes an increase in walking speed, which may in fact lead to more stable gait [21]. Alternatively, the increase in walking speed with lower limb muscle fatigue may be a strategy to cover the short distance walked in this study in as little time as possible $[4,8]$. However, higher walking speed decreases the time available to acquire information and to integrate sensory information [22], which may increase the risk of falling, especially in patients with PD who have an impaired sensory integration capacity [23]. This might lessen the pronounced changes observed in the PD group. Gait changes observed also have consequences for balance in the medio-lateral direction; healthy subjects enlarged their base of support by increasing step width, which did not occur in the PD group. The lack of change in step width of the PD group may suggest a robust system in patients with PD due to the difficulty in performing an adjustment - stiffness strategy [22]. Furthermore, patients with PD present a degradation in sensorimotor integration and sensory feedback $[25,26]$, which may be associated with deficits in perception of fatigue $[2,24]$ and consequently gait adjustments to improve safety. Another alternative explanation for the less pronounced changes in patients with PD may be that the central activation of their muscles in the fatigue protocol was insufficient to cause muscle overload and induce metabolic fatigue [5]. However, the task was standardized and no interaction effect of group and fatigue was found on the maximum voluntary isometric contraction force.
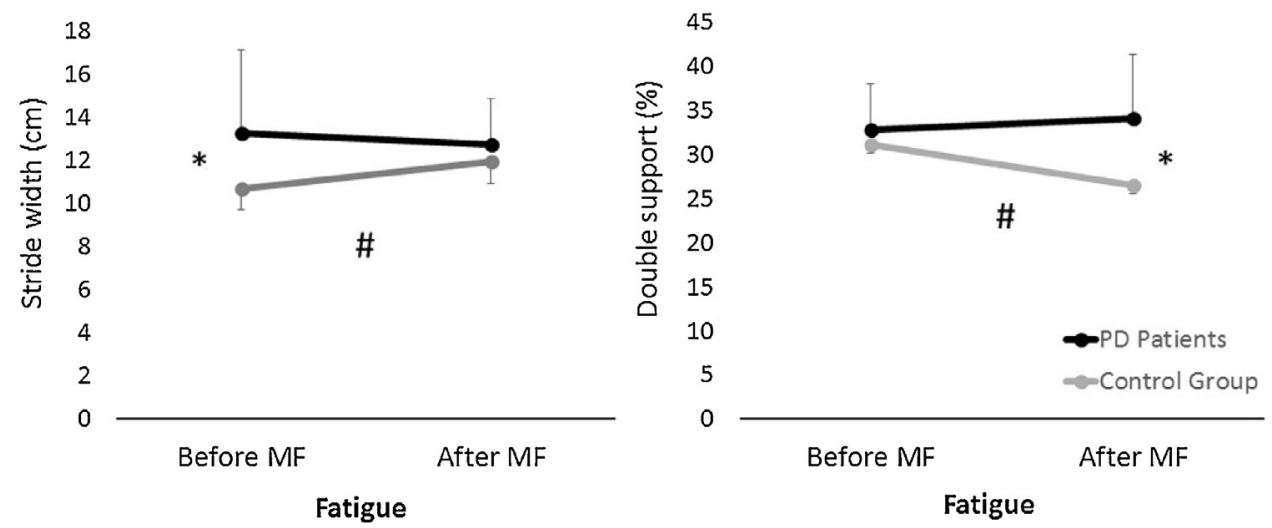

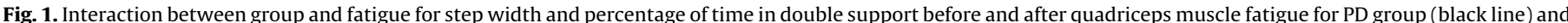

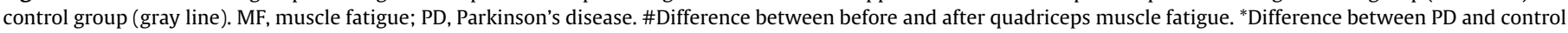
group. 
Physical activity level did have a main effect on gait parameters, indicating a more vigorous and 'healthy' gait pattern in the more active sub-groups. Physical activity improves muscle condition [24] and the negative perception of fatigue [26], which may underlie the differences in gait between the active and inactive sub-groups. For the active PD group, these effects may be important, as they would counteract bradicinesia and hypometria [10]. However, given the crosssectional nature we cannot establish the direction of the causality. Importantly, the physical activity level did not interact with lower limb muscle fatigue. This may be because the gait task in this study did not pose large demand on the locomotor system, while more complex tasks, such as obstacle crossing during walking [27], might reveal such interactions, especially for patients with $\mathrm{PD}$, who experience more difficulty with obstacle crossing [23].

Although the results of this study are consistent and relevant, this study has certain limitations that should be addressed: (i) the lack of a group which was not induced to muscle fatigue, but performed the same protocol without muscle fatigue was a limitation of the study. The lack of inclusion of a control group may hinder some statements about if the changes occur due to the fatigue protocol or another uncontrolled factor (i.e., the time duration of experimental procedures or learning effect). However, similar results were found in different populations $[4,5,8]$ and environments [28], even after 20 min of rest [29], which seems to indicate consistent results regarding the effects of muscle fatigue on gait parameters; (ii) the task to induce lower limb muscle fatigue was the sit-to-stand task, which requires substantial activity of the quadriceps femoris muscles but also of other lower limb muscles. Patients with PD present muscle weakness and an exacerbated perception of fatigue [6], which may decrease the time in the muscle fatigue protocol and limit muscle fatigue induction in this group. However, the maximum voluntary isometric contraction, the indicator of muscle fatigue $[4,8]$, decreased as much in the PD group as in the healthy group; (iii) the muscle fatigue protocol could be made more specific for walking, for example, walking until exhaustion. While this would improve the ecological validity, the time to induce muscle fatigue would increase considerably and this might interfere with timedependent effects of medication in the PD group [30]; (iv) the MBQOA, used to divide the participants into active and inactive sub-groups, does not evaluate all potentially relevant tasks (such as occupational tasks) and may not discriminate physical activity levels adequately; (v) we used a relatively small sample of participants. Considering these limitations, generalization should be performed with care.

We concluded that lower limb muscle fatigue affects the gait parameters of patients with PD and neurologically healthy matched-individuals. Both groups demonstrated gait changes that could be interpreted as seeking to improve balance and safety after lower limb muscle fatigue. However, these adjustments were less pronounced in patients with PD. Moreover, physical activity level did not affect the gait changes occurring after lower limb muscle fatigue, either in patients with PD or in healthy individuals. The findings of this study add to the knowledge about walking in patients with PD, especially related to physical activity and muscle fatigue. Physical activity is an important aspect to improve gait in patients with PD, counteracting the advance of the disease, although having no interaction with lower limb muscle fatigue. However, clinically, patients with PD presented a robust system, which was characterized by difficulty in performing adjustments after lower limb muscle fatigue. Thus, our results can help in developing strategies dealing with the effects of lower limb muscle fatigue on gait parameters in patients with PD.

\section{Conflict of interest statement}

None declared.

\section{References}

[1] Takakusaki K, Saitoh HK, Harada H, Kashiwayanagi M. Role of basal gangliabrainstem pathways in the control of motor behaviors. Neurosci. Res. 2004:50:137-51.

[2] Santos PCR, Barbieri FA, Orcioli-Silva D, Simieli L, Gobbi LTB. Effects of physical activity levels on fatigue perception in patients with Parkinson's disease and neurologically healthy individuals. Health 2014;6:1-6.

[3] Pavese N, Metta V, Bose SK, Chaudhuri KR, Brooks DJ. Fatigue in Parkinson's disease is linked to striatal and limbic serotonergic dysfunction. Brain 2013;133(11):3434-43.

[4] Barbieri FA, Santos PCR, Simieli L, Orcioli-Silva D, van Dieën JH, Gobbi LTB. Interactions of age and leg muscle fatigue on unobstructed walking and obstacle crossing. Gait Posture 2014;39:985-90.

[5] Stevens-Lapsley J, Kluger BM, Schenkman M. Quadriceps muscle weakness, activation deficits, and fatigue with Parkinson disease. Neurorehabil. Neural Repair 2012;26(5):33-41.

[6] Haskell WL, Lee I-M, Pate RR, et al. Physical activity and public health: updated recommendation for adults from the American College of Sports Medicine and the American Heart Association. Circulation 2007;116: 1081-93.

[7] Katsiaras A, Newman AB, Kriska A, et al. Skeletal muscle fatigue, strength, and quality in the elderly: the Health ABC Study. J. Appl. Physiol. 2005;99: 210-6.

[8] Barbieri FA, Santos PC, Vitório R, Van Dieën JH, Gobbi LTB. Effect of muscle fatigue and physical activity level in motor control of the gait of young adults. Gait Posture 2013;38(4):702-7.

[9] Orcioli-Silva D, Simieli L, Barbieri FA, Rinaldi NM, Vitório R, Gobbi LTB. Effects of a multimodal exercise program on the functional capacity of Parkinson disease patients considering disease severity and gender. Motriz. Rev. Ed. Fis. 2014;20:1-4.

[10] Vitório R, Lirani-Silva E, Barbieri FA, et al. Effects of 6-month, multimodal exercise program on clinical and gait parameters of patients with idiopathic Parkinson's disease: a pilot study. ISRN Neurol. 2011;1-7.

[11] Hughes AJ, Daniel SE, Kilford L, Lees AJ. Accuracy of clinical diagnosis of idiopathic Parkinson's disease: a clinico-pathological study of 100 cases. J. Neurol. Neurosurg. Psychiatry 1992;55:181-4.

[12] Hoehn MM, Yahr MD. Parkinsonism: onset, progression and mortality. Neurology 1967; 17:427-42.

[13] Voorrips LE, Ravelli AC, Dongelmans PC, Deurenberg P, Van Staveren WA. A physical activity questionnaire for the elderly. Med. Sci. Sports Exerc. 1991;23(8):974-9.

[14] Nascimento CMC, Gobbi S, Hirayama MS, Brazão MC. Nível de atividade física e as principais barreiras percebidas por idosos de Rio Claro. REVDEF 2008;19(1):109-18

[15] Fahn S, Elton R, Members of the UPDRS Development Committee. The unified Parkinson's disease rating scale. In: Fahn S, Marsden CD, Calne DB, Goldstein M, editors. Recent Developments in Parkinson's Disease, vol. 2. Florham Park, NJ: McMellam Health Care Information; 1987. p. 153-63.

[16] Brucki SMD, Nitrini R, Caramelli P, Bertolucci PHF, Okamoto IH. Sugestões para o uso do mini-exame do estado mental no Brasil. Arq. Neuropsiquiatr. 2003;61(3-B):777-81

[17] Hof AL, Gazendam MG, Sinke WE. The condition for dynamic stability. J Biomech. 2005;38:1-8.

[18] Hof AL, van Bockel RM, Schoppen T, Postema K. Control of lateral balance in walking: experimental findings in normal subjects and above-knee amputees. Gait Posture 2007;25:250-8.

[19] Hak L, Houdijk H, Steenbrink F, et al. Speeding up or slowing down? Gait adaptations to preserve gait stability in response to balance perturbations. Gait Posture 2012;36:260-4.

[20] Carpenter MG, Allum JHJ, Honegger F, Adkin AL, Bloem BR. Postural abnormalities to multidirectional stance in Parkinson's disease. J. Neurol. Neurosurg. Psychiatry 2004;75:1245-54.

[21] Bruijn SM, van Dieen JH, Meijer OG, Beek PJ. Is slow walking more stable? J. Biomech. 2009;42:1506-12.

[22] Lewis GN, Byblow WD. Altered sensorimotor integration in Parkinson's disease. Brain 2002;125(9):2089-99.

[23] Vitório R, Lirani-Silva E, Barbieri FA, Raile V, Stella F, Gobbi LTB. Influence of visual feedback sampling on obstacle crossing behavior in people with Parkinson's disease. Gait Posture 2013;37(2):330-4.

[24] Solla P, Cannas A, Mulas CS, et al. Association between fatigue and other motor and non-motor symptoms in Parkinson's disease patients. J. Neurol. 2014;261(2):382-91.

[25] Nelson ME, Rejeski WJ, Blair SN, et al. Physical activity and public health in older adults: recommendation from the American College of Sports Medicine and the American Heart Association. Circulation 2007;116: 1094-105.

[26] Garber CE, Friedman JH. Effects of fatigue on physical activity and function in patients with Parkinson's disease. Neurology 2003;60(7):1119-24. 
[27] Lowrey CR, Watson A, Ann L. Age-related changes in avoidance strategies when negotiating single and multiple obstacles. Exp. Brain Res. 2007;182: 289-99.

[28] Barbieri FA, Lee YJ, Gobbi LT, Pijnappels M, Van Dieën JH. The effect of muscle fatigue on the last stride before stepping down a curb. Gait Posture 2013:37(4):542-6.
[29] Barbieri FA, Beretta SS, Pereira VAI, et al. Recovery of gait after quadriceps muscle fatigue. Gait Posture 2015;43:270-4.

[30] Kempster PA, Frankel JP, Bovingdon M, Webster R, Lees AJ, Stern GM. Levodopa peripheral pharmacokinetics and duration of motor response in Parkinson's disease. J. Neurol. Neurosurg. Psychiatry 1989;52:718-23. 